\title{
Alignment of polymer based magnetic composites in magnetic field
}

\author{
A.V. Kukhta ${ }^{\mathrm{a}, \mathrm{b}}$, P.P. Kuzhir ${ }^{\mathrm{a}, \mathrm{b}}$, S.A. Maksimenko ${ }^{\mathrm{a}, \mathrm{b}}$, S.A. Vorobyova ${ }^{\mathrm{c}}$, I.N. Kukhta ${ }^{\mathrm{d}}$, \\ A.E. Pochtenny ${ }^{\mathrm{e}}$, M.I. Taoubi ${ }^{\mathrm{f}}$, S. Bistarelli ${ }^{\mathrm{g}}$, S. Bellucci ${ }^{\mathrm{g}, *}$

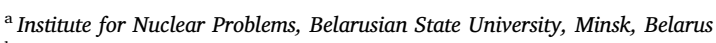 \\ ${ }^{\mathrm{b}}$ Tomsk State University, Tomsk, Russia \\ ${ }^{\mathrm{c}}$ Research Institute for Physical and Chemical Problems, Belarusian State University, Minsk, Belarus \\ ${ }^{\mathrm{d}}$ Institute of Chemistry of New Materials, National Academy of Sciences of Belarus, Minsk, Belarus \\ ${ }^{\mathrm{e}}$ Belarusian State University of Technology, Minsk, Belarus \\ ${ }^{\mathrm{f}}$ Faculty of Sciences No5, Lebanese University, Elnabatieh, Lebanon \\ ${ }^{\mathrm{g}}$ National Institute of Nuclear Physics, Frascati National Laboratory, Frascati, Italy
}

A R T I C L E I N F O

\section{Keywords:}

Nanocomposites

PEDOT:PSS

GNP

$\mathrm{Fe}_{3} \mathrm{O}_{4}$

Alignment

\begin{abstract}
A B S T R A C T
Composite films made of graphene nanoplatelets (GNP) and magnetite $\mathrm{Fe}_{3} \mathrm{O}_{4}$ nanoparticles have been prepared with and without magnetic field. Poly(3,4-ethylenedioxythiophene):polystyrenesulfonic acid (PEDOT:PSS) was used as a stabilizing polymer matrix. Film morphology was found to depend strongly on the presence of magnetic field, i.e. more porous structure of GNP- $\mathrm{Fe}_{3} \mathrm{O}_{4}$ is formed in magnetic field. Thin films formed without magnetic field have activation character of conductivity, while thin films formed in magnetic field have metallic conductivity. Thus, the magnetic field acting during film formation in these composites results in semiconductormetal transition. DFT calculations show that at low distance between GNP surface and nanoparticle strong interaction between them can be observed.
\end{abstract}

\section{Introduction}

Graphene and graphene nanoplatelets (GNP) are the most studied materials during the last decade owing to their outstanding properties and manifold applications [1,2]. Alignment of the graphene nanoplatelets is very attractive for many electronic applications because of the active surface increase. The efficient growth of vertically oriented graphene nanosheets on nickel surface has been recently achieved during chemical vapor deposition [3]. However, this tool is very costly and time consuming, as well as surface limiting. Application of the stacking polymers $[4,5]$ is known to result in some GNP alignment, but along the substrate surface. The most attractive configuration is GNP aligned perpendicularly to the substrate, because it can result in the highest increase of the active surface. The electric field [6] is useful for the GNP alignment, however it requires rather high electric fields damaging the structure. The usage of special groups at GNP edges can align GNP perpendicularly to the surface using Langmuir-Blodgett approach [7]. However, such groups are strongly insulating what is a drawback for electronic applications. One of the ways of alignment of such materials can be the formation of the films in the magnetic field. For this purpose, composites based on graphene nanoplatelets covered with magnetic nanoparticles are proposed. These nanoparticles are known to align easily within magnetic field lines in magnetic liquids [8] similarly to magnetic microparticles. The decoration of GNP by metal nanoparticles was found to improve the film conductivity and stability [9]. Composites based on nanoparticles made from magnetic metals are promising for various potential applications such as spin-polarized devices, carriers for drug delivery, magnetic recording media, high-frequency applications, magneto-optical storage, interference suppression, biomedical sensing, etc. [10,11]. Magnetite, $\mathrm{Fe}_{3} \mathrm{O}_{4}$, is an attractive material for anodes in manifold electronic devices and spintronics due to its high theoretical capacity, high density, low cost, low toxicity, and outstanding magnetic properties [12]. Thus, the usage of these two materials can combine their properties. This approach results in the improvement of thermal conductivity [13]. The graphene alignment in such a composite can be reached even at low magnetic field [14]. Typically, for the synthesis of graphene- $\mathrm{Fe}_{3} \mathrm{O}_{4}$ composites the graphene oxide is used [12,15]. In this case $\mathrm{Fe}_{3} \mathrm{O}_{4}$ nanoparticles are chemically attached to the reduced graphene oxide by means of chemical reaction. However, conductivity of graphene oxide is low [1].

In this work we synthesized polymer composites based on conducting polymer filled with GNP covered by $\mathrm{Fe}_{3} \mathrm{O}_{4}$ nanoparticles [16]. Poly(3,4-ethylenedioxythiophene):polystyrenesulfonic acid (PEDOT:PSS) was used as a conducting polymer matrix. PEDOT:PSS is

\footnotetext{
* Corresponding author.

E-mail address: bellucci@lnf.infn.it (S. Bellucci).
} 
attractive to be used as a matrix owing to good interaction with GNP. Though, any polymer can be used for this purpose (for example, polyaniline $[17,18])$, PEDOT:PSS is attractive owing to its good interaction with GNP. Thin films of this composite were grown in magnetic field, and morphology and electrophysical properties have been studied.

\section{Experimental}

GNP are few layer graphene nanosheets produced by microwave (800 W) exfoliation of ASBURY expandable graphite [19-23] followed by a sonication in ultrasonic bath in water-alcohol solution for size reduction, and further enriched by centrifugation. We used this "green" water-isopropanol solvent, because water-ethanol gives good enough results for graphene dispersion [24], having higher boiling point making difficult a solvent evaporation during nanoparticle synthesis, and compatible for NP synthesis. Isopropanol can be easily mixed with water. The volume ratio of water:isopropanol is $4: 1$.

The synthesis of $\mathrm{Fe}_{3} \mathrm{O}_{4}$ nanoparticles was made in the previous manner [25] directly in GNP suspension by the following way. At the first stage $14.9 \mathrm{mg}$ of iron (III) chloride hexahydrate $\mathrm{FeCl}_{3} \cdot 6 \mathrm{H}_{2} \mathrm{O}$ were dissolved in $5 \mathrm{ml}$ of distilled water and $8.5 \mathrm{mg}$ of iron (II) sulfate heptahydrate $\mathrm{FeSO}_{4} \cdot 7 \mathrm{H}_{2} \mathrm{O}$ were dissolved in $5 \mathrm{ml}$ of distilled water with 1-2 drops of concentrated sulfuric acid (92\%). Separately the graphene (GNP) dispersion were prepared by adding of $6.4 \mathrm{mg}$ of GNP to $8 \mathrm{ml}$ of isopropyl alcohol. This suspension was ultrasonicated during $45 \mathrm{~min}$. Then $0067 \mathrm{ml}$ of the concentrated ammonium hydroxide (25\%) were added to GNP suspension. Thereafter the irons salt solutions, obtained in the first stage, were added slowly to GNP suspension, containing ammonium hydroxide. The prepared $\mathrm{Fe}_{3} \mathrm{O}_{4}$-GNP suspension was carefully washed several times by water-isopropanol solution for removing the excess of reaction products and magnetite NP that was not attached to graphene. Polymer composites have been obtained by mixing the calculated amounts of polymer and graphene-magnetite solution. All reagents were used as received without any additional purification. Water solution of PEDOT:PSS (low conduction grade) was purchased from Sigma Aldrich.

We prepared composite films made from GNP and magnetite nanoparticles and formed in magnetic field and without it. $\mathrm{Fe}_{3} \mathrm{O}_{4}$ nanoparticles represent a magnetic liquid in water-isopropanol solution. This liquid is very sensitive to magnetic field resulting in high alignment of iron oxide nanoparticles. The composite solution was dropcasted on Si or quartz substrate, and polycor substrate with $5 \mathrm{~mm}$ long $\mathrm{Ni}$ electrodes separated by $100 \mu \mathrm{m}$ distance, followed by heating under vacuum at a temperature of $100{ }^{\circ} \mathrm{C}$ for $30 \mathrm{~min}$. Before deposition, the substrates were ultrasonically cleaned in acetone for $15 \mathrm{~min}$, dried in a nitrogen flow, and then irradiated with oxygen plasma for $15 \mathrm{~min}$ to remove any organic contamination. Thin films are formed by drying in air in magnetic field $0.15 \mathrm{Tl}$ (perpendicularly to the film surface) or without it. The film thickness was $400-500 \mathrm{~nm}$. In order to measure the thickness, the films were scratched to expose the substrate and a Solver P47 atomic force microscopy in tapping mode was used to scan from the film top to the exposed substrate surface. Three independent procedures of synthesis, film formation and characterization measurements have been carried out, yielding practically the same results.

The morphology of the dispersed phase was examined using a LEO$1455 \mathrm{VP}$ scanning electron microscope with a resolution of $1 \mathrm{~nm}$ at $100 \mathrm{kV}$ and a LEO-906 transmission electron microscope (TEM). The samples for TEM measurements were prepared by dropcasting the sonicated dispersion of the corresponding material in water-propanol solution onto 600 mesh copper grids coated with lacey carbon and dried at room temperature for $3 \mathrm{~h}$. Elemental composition has been determined with X-ray spectral analyzer RONTEG.

The conductivity temperature dependences were studied by means of cyclic thermo-desorption [26] in $10^{-2} \mathrm{~Pa}$ vacuum). The sample is heated up to a certain temperature resulting in the decrease of the adsorbed oxygen in the film, up to some fixed level. Then the sample is cooled with the same concentration of the adsorbed oxygen, and the conductivity temperature dependence is measured during this process. Then this sample is heated again up to a higher temperature, and measurement is repeated at a lower concentration of the adsorbed oxygen. As a result, we obtain a set of conductivity temperature dependences with decreasing adsorbed oxygen in the film in every subsequent heating-cooling cycle.

Density functional theory (DFT) [27] was applied to simulate the interaction between magnetite NP and the first layers of GNP, as well as the spin distribution in the system. Calculations were made in the framework of the quantum chemical Gaussian-09 Program package [28]. Magnetite NP was approximated by the simplest molecule structure consisting of 3 iron atoms and 4 oxygen atoms. GNP was approximated by a rectangular honeycomb structure built from benzene rings with values of $\mathrm{C}-\mathrm{C}$ bonds replaced with experimental data [29].

\section{Results and discussion}

Graphene nanoplatelets have typical width of $10-20 \mu \mathrm{m}$ and thickness up to $3 \mathrm{~nm}[6,19]$. It was shown [6] that their Raman spectrum does not contain the D peak, thus pointing out the absence of chemical defects.

Fig. 1a shows a TEM picture and the diameter distribution (inset) of iron oxide NP synthesized without GNP. The histogram corroborates the narrow size distribution with maximum number of particles around $7 \pm 1.4 \mathrm{~nm}$, along with larger particles up to $15.9 \mathrm{~nm}$ and the smallest particles of $3.5 \mathrm{~nm}$. XRD spectrum contains peaks at the angles 41.46, $35.15,50.54,67.4$ and $74.25^{\circ}$ confirming the formation of $\mathrm{Fe}_{3} \mathrm{O}_{4}$ phase [25]. The obtained suspension is highly sensitive to the presence of magnetic field. When we prepared a thin film of pure iron oxide
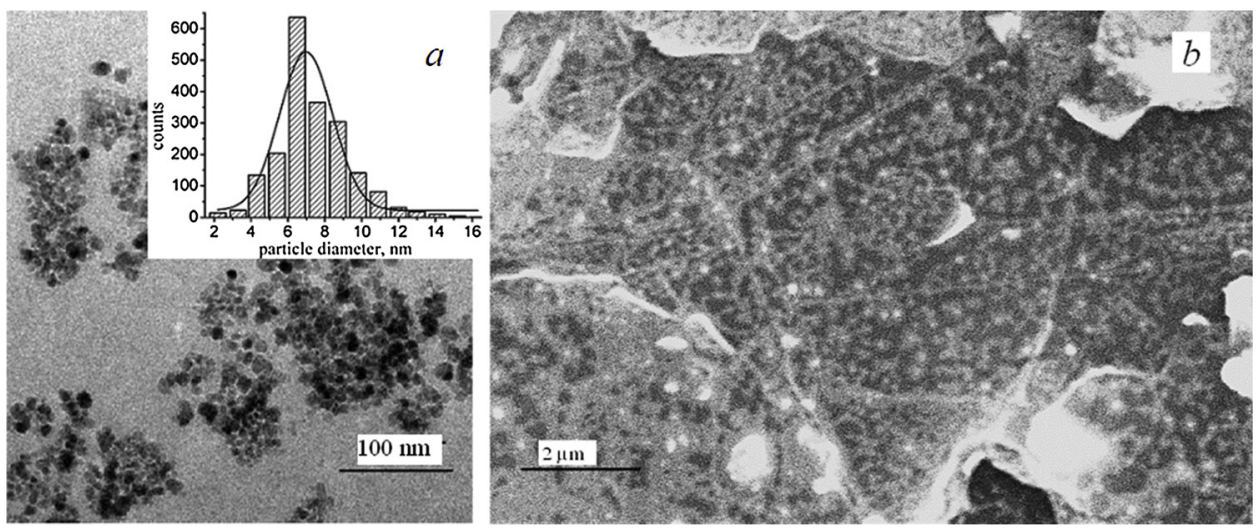

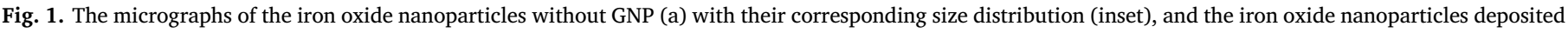
on GNP (b). 


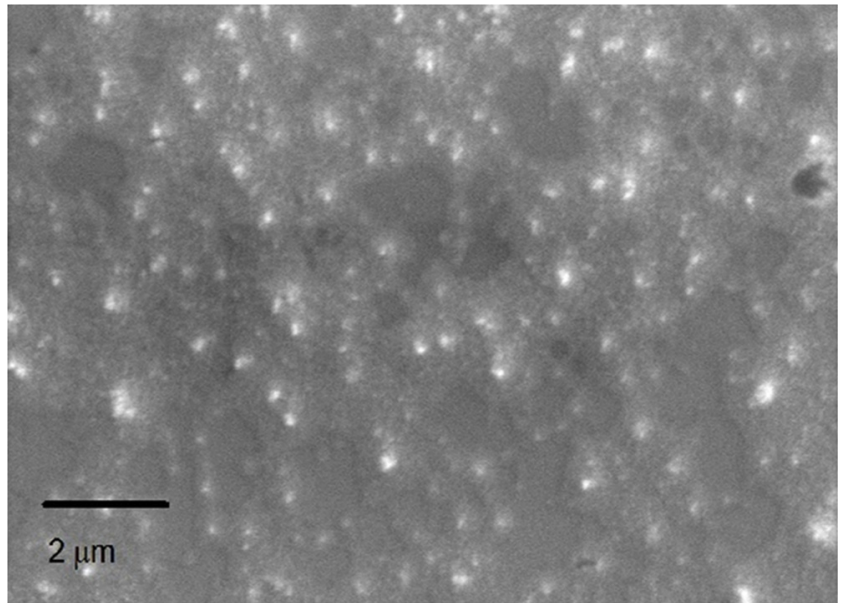

Fig. 2. The alignment of the iron oxide nanoparticles in a magnetic field.

nanoparticles in a magnetic field (perpendicularly to the substrate surface), these nanoparticles form an ordered structure (see two-dimensional distribution perpendicularly to the magnetic field in Fig. 2). It can be supposed that these bright points represent needles consisting of $\mathrm{Fe}_{3} \mathrm{O}_{4}$ nanoparticles attracted to each other in magnetic field. These nanoparticles are easily attached to GNP surface in the same reaction in the presence of GNP suspension. The high microscope resolution image (Fig. 1b) showed that the corresponding nanoparticles are physically adsorbed on the graphene surface randomly and homogeneously enough, similarly to copper nanoparticles [6]. The final GNP-magnetite suspension is also very sensitive to the magnetic field. The obtained GNP: $\mathrm{Fe}_{3} \mathrm{O}_{4}$ weight ratio was $60 \%: 40 \%$. No visible changes in Raman spectrum have been observed.

Then we prepared thin films made from GNP covered with iron oxide nanoparticles forming them in the magnetic field and without it. Fig. 3 illustrates the micrographs of both films. It can be seen that the thin film formed in the magnetic field has GNP located not strictly perpendicularly to the surface, as expected. However, this film has a more porous structure and active surface as compared to the film without the field. This fact confirms the strong effect of the magnetic field on the formation of this composite morphology. It can be supposed that magnetite nanoparticles on GNP surface tend to be attracted to each other in magnetic field similar to that in pure nanoparticle suspension. In the volume they cannot be attached very close forming nanoparticle needles, it results in the formation of a more porous film morphology.

It was found that the magnetic field applied to samples during film formation also affects strongly the electric properties of these films. The conductivity temperature dependences are presented below for thin films based on graphene (Fig. 4) and polymer composite based on graphene with iron oxide nanoparticles, formed in the magnetic field and without it (Fig. 5). Note that composite films made from GNP covered with iron oxide NP are not enough stable mechanically. Thus, PEDOT:PSS polymer matrix is used for the stabilization of the film structure during heating-cooling processes. The weight ratio of polymer:filler was $1: 1$.

It can be seen (Fig. 4) that the resistance of the graphene films is growing with the temperature increase with a slight super-linearity. Such a behavior is typical for semimetals containing admixtures acting as centers of scattering of charge carriers. During the oxygen desorption the resistance of the graphene film decreases that can be caused by decreasing of the concentration of the scattering centers and increasing of the mobility of charge carriers. The curves obtained for thin films made of graphene are linear in conductivity-temperature coordinates.

The temperature dependences of the conductivity of thin films of polymer composites based on graphene with iron oxide nanoparticles,
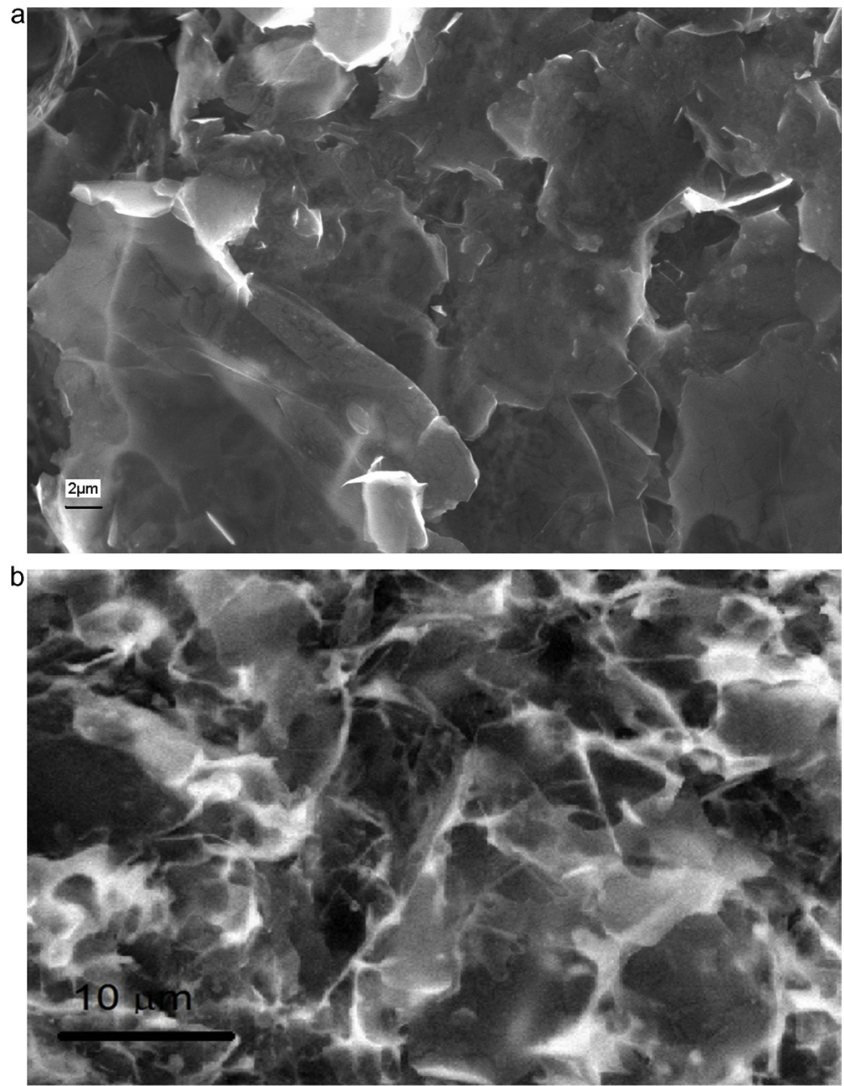

Fig. 3. The morphology of the films made from GNP covered with iron oxide nanoparticles and formed without magnetic field (a) and in a magnetic field perpendicularly to the substrate surface (b)

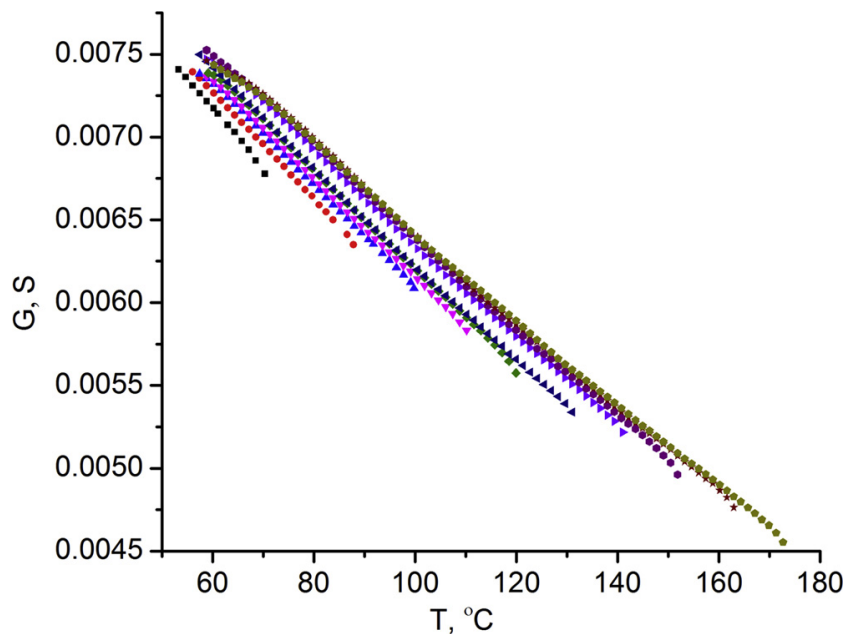

Fig. 4. Temperature conductivity depencences for pure GNP film made for different highest heating temperatures.

formed in magnetic field and without it, show a different behaviour (compare Fig. $5 \mathrm{a}$ and b). The thickness and dimensions of the film in both cases are approximately the same. Graphene - iron oxide composite films formed in the magnetic field show temperature conductivity dependences similar to that of graphene with metallic conductivity. As opposite, graphene - iron oxide composite films formed without magnetic field have a semiconductor activation character of the conductivity with not essential temperature changes of activation energy in the range of $0.029-0.034 \mathrm{eV}$. The conductivity value at the beginning of heating was twice smaller, but it became practically the 

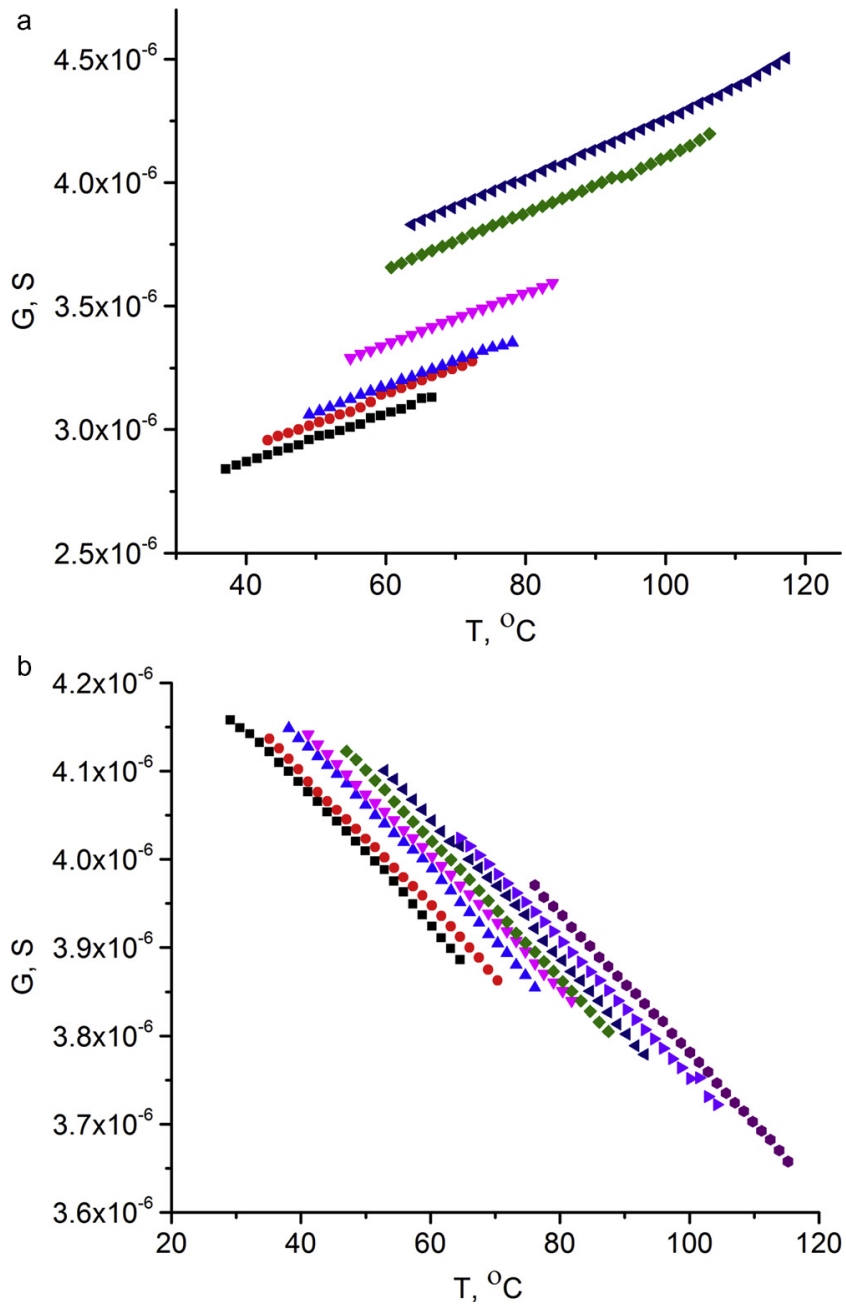

Fig. 5. Temperature conductivity dependences for thin films of polymer GNP$\mathrm{Fe}_{3} \mathrm{O}_{4}$ composite formed without magnetic field (a) and in magnetic field (b), made for different highest heating temperatures.

same after heating. Thus, the magnetic field acting during graphene$\mathrm{Fe}_{3} \mathrm{O}_{4}$ film formation results in the dielectric-metal transition. It should be noticed that, comparing the spread of the temperature dependences there is considerable difference in the value of conductivity, owing to oxygen adsorption in pure GNP and composite films, pointing out at higher porosity of the latter film.

The conductivity of GNP is metallic, and the conductivity of magnetite is semiconducting. Thus, it can be expected that morphology changing in GNP-magnetite composite can result in the observed phenomenon. The magnetic field attracts nanoparticles closer to each other and improves contacts between nanoplatelets.

The observed behavior can apparently depend on the interaction between GNP and $\mathrm{Fe}_{3} \mathrm{O}_{4}$ nanoparticles. To verify this assumption, DFT simulation of this system was applied using Gaussian-09 Program package. Calculations were made to minimize the conformational energy of the simulated structure. We choose to describe only one-layer GNP in the simulation, because we suppose that in this case the interaction with nanoparticle can result in stronger structure deformation than in the case of multilayered GNP, owing to $\pi$ - $\pi$-stacking of graphene layers. According to simulation, the interaction between counterparts depends on the GNP-NP distance. At higher distance, weak interaction is observed (Fig. 6a) that can be noticed in some electronic density redistribution in GNP and nanoparticles (not presented). This energy redistribution, however, is not enough to result in conductivity type changes. Note that non interacting nanoparticles are easily
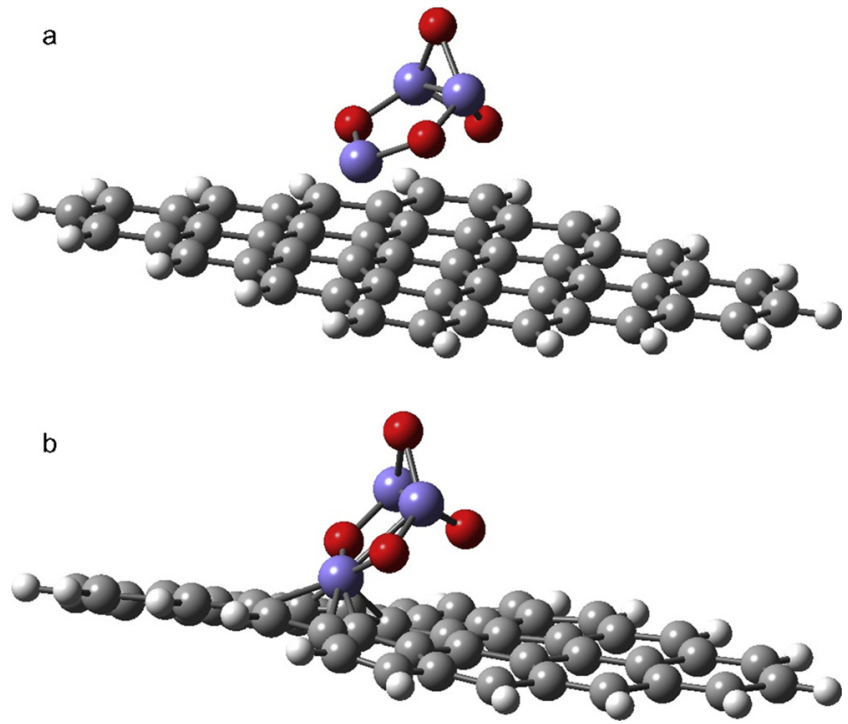

Fig. 6. The simulation of interaction between GNP and magnetite molecule at higher (a) and lower (b) distance.

removed from the composite solution during washing procedure. When the distance is low enough, the formation of chemical bonds with GNP surface skewing is observed (Fig. 6b). Essential interaction between $\mathrm{Fe}_{3} \mathrm{O}_{4}$ and graphene is confirmed by other calculations [30,31] and experimental data [32]. Calculations [33] showed that magnetic field aff ;ects strongly the electron density of molecules. The effect of charge transfer process can be quantified in terms of shifting of Fermi level of these components [34].

In order to find the most reliable explanation the further experiments are required.

\section{Conclusions}

We prepared composite films made from GNP and magnetite $\mathrm{Fe}_{3} \mathrm{O}_{4}$ nanoparticles, stabilized by PEDOT:PSS polymer, and formed in magnetic field. It was found that the magnetic field applied to samples during film formation strongly affects the morphology and electric properties of these films. GNP covered with magnetite nanoparticles tend to align along the magnetic field and form more porous structure. Thin films formed without magnetic field have activation character of conductivity, while those formed in magnetic field has metallic conductivity. The magnetic field attracts nanoparticles closer to each other and improves contacts between nanoplatelets. The possible mechanisms of these transformations are discussed in terms of the increase of interaction between counterparts in magnetic field. To verify this assumption, DFT simulation of this system was applied using Gaussian-09 Program package. According to simulation, the interaction between counterparts depends on the GNP-NP distance. At higher distance, weak interaction is observed as a noticeable electronic density redistribution in GNP and nanoparticles. When the distance is low enough, the formation of chemical bonds with GNP surface skewing is observed. The proposed facile wet-processing technology to obtain vertically aligned graphene nanosheets enable low-cost and large area processing for manifold applications.

\section{Declaration of Competing Interest}

The Authors declare no conflict of interest.

\section{Acknowledgments}

This work is supported by the EU FP7-PEOPLE-2013-IRSES-610875 
NAmiceMC, the EU H2020 project GrapheneCore1 - Grant Agreement AMD-696656-4, H2020 RISE 734164 Graphene 3D. PPK and SEM are also thankful for support by Tomsk State University Competitiveness Improvement Program.

\section{References}

[1] K.S. Novoselov, V.I. Fal'ko, L. Colombo, P.R. Gellert, M.G. Schwab, K. Kim, A roadmap for graphene, Nature 490 (2012) 192-200, https://doi.org/10.1038/ nature11458.

[2] X. Yu, H. Cheng, M. Zhang, Y. Zhao, L. Qu, G. Shi, Graphene-based smart materials, Nat. Rev. Mat. 2 (2017) 17046, https://doi.org/10.1038/natrevmats.2017.46.

[3] Yi Zhang, Luyao Zhang, Chongwu Zhou, Review of chemical vapor deposition of graphene and related applications, Acc. Chem. Res. 46 (2013) 2329-2339, https:// doi.org/10.1021/ar300203n.

[4] Weifeng Zhao, Jie Kong, Hu Liu, Qiang Zhuang, Junwei Gu, Zhanhu Guo, Ultra-high thermally conductive and rapid heat responsive poly(benzobisoxazole) nanocomposites with self-aligned graphene, Nanoscale 8 (2016) 19984-19993, https:// doi.org/10.1039/C6NR06622D.

[5] Yan-Feng Bai, Yong-Fang Zhang, An-Wei Zhou, Hai-Wai Li, Yu Zhang, John H.T. Luong, Hui-Fang Cui, Self-assembly of a thin highly reduced graphene oxide fi $1 \mathrm{~m}$ and its high electrocatalytic activity, Nanotechnology 25 (2014) 405601.

[6] Shuying Wu, Raj B. Ladani, Jin Zhang, E. Bafekrpour, K. Ghorbani, A.P. Mouritz, A.J. Kinloch, C.H. Wang, Aligning multilayer graphene flakes with an external electric field to improve multifunctional properties of epoxy nanocomposites, Carbon 94 (2015) 607-618, https://doi.org/10.1016/j.carbon.2015.07.026.

[7] A. Rodríguez-Serradet, S. Ciftci, A. Mikosch, A.J.C. Kuehne, C.P. de Melo, R. CaoMilán, Vertical assembly of few-layer graphene decorated with iron oxide nanoparticles on gold surfaces, RCS Adv. 6 (2016) 94256-94262, https://doi.org/10. 1039/C6RA15502B.

[8] I. Torres-Díaz, C. Rinaldi, Recent progress in ferrofluids research: novel applications of magnetically controllable and tunable fluids, Soft Matter 10 (2014) 8584-8602, https://doi.org/10.1039/C4SM01308E.

[9] A.V. Kukhta, A.G. Paddubskaya, P.P. Kuzhir, S.A. Maksimenko, S.A. Vorobyova, S. Bistarelli, A. Cataldo, S. Bellucci, Copper nanoparticles decorated graphene nanoplatelets and composites with PEDOT:PSS, Synth. Met. 222 (2016) 192-197, https://doi.org/10.1016/j.synthmet.2016.10.006.

[10] H. Deng, X.L. Li, Q. Peng, X. Wang, J.P. Chen, Y.D. Li, Monodisperse magnetic single-crystal ferrite microspheres, Angew. Chem. Int. Ed. 44 (2005) 2782, https:// doi.org/10.1002/anie.200462551.

[11] O.V. Kharissova, B.O. García, B.I. Kharisov, U.O. Méndez, Magnetic-graphene-based nanocomposites and respective applications, in: Adrian M.T. Silva, S.A.C. Carabineiro (Eds.), Advances in Carbon Nanostructures, 2016, https://doi. org/10.5772/64319.

[12] G. Zhou, D.-W. Wang, F. Li, L. Zhang, N. Li, Z.-S. Wu, L. Wen, G.Q. Lu, H.-M. Cheng, Graphene-wrapped $\mathrm{Fe}_{3} \mathrm{O}_{4}$ anode material with improved reversible capacity and cyclic stability for lithium ion batteries, Chem. Mater. 22 (2010) 5306-5313, https://doi.org/10.1021/cm101532x.

[13] J. Renteria, S. Legedza, R. Salgado, M.P. Balandin, S. Ramirez, M. Saadah, F. Kargar, A.A. Balandin, Magnetically-functionalized self-aligning graphene fillers for highefficiency thermal management applications, Mater. Des. 88 (2015) 214-221, https://doi.org/10.1016/j.matdes.2015.08.135.

[14] S. Wu, J. Zhang, R.B. Ladani, K. Ghorbani, A.P. Mouritz, A.J. Kinloch, C.H. Wang, A novel route for tethering graphene with iron oxide and its magnetic field alignment in polymer nanocomposites, Polymer 97 (2016) 273-284, https://doi.org/10.1016/ j.polymer.2016.05.024.

[15] S. Bhuvaneswari, P.M. Pratheeksha, S. Anandan, D. Rangappa, R. Gopalan, T.N. Rao, Efficient reduced graphene oxide grafted porous $\mathrm{Fe}_{3} \mathrm{O}_{4}$ composite as a high performance anode material for Li-ion batteries, Phys. Chem. Chem. Phys. 16 (2014) 5284-5294, https://doi.org/10.1039/C3CP54778G.

[16] A.V. Kukhta, A.G. Paddubskaya, P.P. Kuzhir, S.A. Maksimenko, S.A. Vorobyova, S. Bellucci, P.K. Khanna, Electroactive polymer based conducting, magnetic, and luminescent triple composites, Adv. Sci. Technol. 97 (2016) 24-29, https://doi.org/ 10.4028/www.scientific.net/AST.97.24.

[17] Panbo Liu, Ying Huang, Jing Yan, Yang Zhao, Magnetic graphene@PANI@porous TiO2 ternary composites for high-performance electromagnetic wave absorption, J.
Mat. Chem. C 4 (2016) 6362-6370, https://doi.org/10.1039/c6tc01718e.

[18] P. Liu, J. Yan, X. Gao, Y. Huang, Y. Zhang, Construction of layer-by-layer sandwiched graphene/polyaniline nanorods/carbon nanotubes heterostructures for high performance supercapacitors, Electrochim. Acta 272 (2018) 77-87, https://doi. org /10.1016/j.electacta.2018.03.198.

[19] A. Dabrowska, S. Bellucci, A. Cataldo, F. Micciulla, A. Huczko, Nanocomposites of epoxy resin with graphene nanoplates and exfoliated graphite: Synthesis and electrical properties, Phys. Status Solidi B 251 (2014) 2599-2602, https://doi.org/ $10.1002 /$ pssb. 201451175.

[20] L. Pierantoni, D. Mencarelli, M. Bozzi, R. Moro, S. Bellucci, Graphene-based Electronically Tuneable Microstrip Attenuator, Nanomater. Nanotechnol. 4 (2014) 1-6, https://doi.org/10.5772/58758.

[21] M. Bozzi, L. Pierantoni, S. Bellucci, Applications of graphene at microwave frequencies, Radioengineering 24 (2015) 661-669, https://doi.org/10.13164/re. 2015.0661.

[22] L. Pierantoni, D. Mencarelli, M. Bozzi, R. Moro, S. Moscato, L. Perregrini, F. Micciulla, A. Cataldo, S. Bellucci, Broadband microwave attenuator based on few layer graphene flakes, IEEE Trans. Microw. Theory Tech. 63 (2015) 2491-2497, https://doi.org/10.1109/TMTT.2015.2441062.

[23] A. Maffucci, F. Micciulla, A. Cataldo, G. Miano, S. Bellucci, Bottom-up realization and electrical characterization of a graphene-based device, Nanotechnology 27 (2016) 095204, , https://doi.org/10.1088/0957-4484/27/9/095204.

[24] W.-W. Liu, B.-Y. Xia, X.-X. Wang, J.-N. Wang, Exfoliation and dispersion of graphene in ethanol-water mixtures, Front. Mater. Sci. 6 (2012) 176-182, https://doi. org/10.1007/s11706-012-0166-4.

[25] A.I. Lesnikovich, T.M. Shunkevich, V.N. Naumenko, S.A. Vorobyova, M.V. Baikov, Dispersity of magnetite in magnetic liquid and the interaction with a surfactant, J. Magn. Magn. Mat. 85 (1990) 14-16.

[26] A.V. Kukhta, E.E. Kolesnik, I.N. Kukhta, A.E. Pochtenny, V.K. Dolgiy, G.A. Mousdis, N. Psaroudakis, Optical and electrophysical properties of sulfur containing metal free phthalocyanine, Synth. Met. 160 (2010) 2361-2365, https://doi.org/10.1016/ j.synthmet.2010.09.012

[27] F. Weigend, M. Haser, H. Patzelt, R. Ahlrichs, RI-MP2: optimized auxiliary basis sets and demonstration of efficiency, Chem. Phys. Lett. 294 (1998) 143-152, https:// doi.org/10.1016/S0009-2614(98)00862-8.

[28] M.J. Frisch, G.W. Trucks, H.B. Schlegel, G.E. Scuseria, M.A. Robb, J.R. Cheeseman, G. Scalmani, V. Barone, B. Mennucci, G.A. Petersson, H. Nakatsuji, M. Caricato, X. Li, H.P. Hratchian, A.F. Izmaylov, J. Bloino, G. Zheng, J.L. Sonnenberg, M. Hada, M. Ehara, K. Toyota, R. Fukuda, J. Hasegawa, M. Ishida, T. Nakajima, Y. Honda, O. Kitao, H. Nakai, T. Vreven, J.A. Montgomery Jr., J.E. Peralta, F. Ogliaro, M. Bearpark, J.J. Heyd, E. Brothers, K.N. Kudin, V.N. Staroverov, R. Kobayashi, J. Normand, K. Raghavachari, A. Rendell, J.C. Burant, S.S. Iyengar, J. Tomasi, M. Cossi, N. Rega, J.M. Millam, M. Klene, J.E. Knox, J.B. Cross, V. Bakken, C. Adamo, J. Jaramillo, R. Gomperts, R.E. Stratmann, O. Yazyev, A.J. Austin, R. Cammi, C. Pomelli, J.W. Ochterski, R.L. Martin, K. Morokuma, V.G. Zakrzewski, G.A. Voth, P. Salvador, J.J. Dannenberg, S. Dapprich, A.D. Daniels, O. Farkas, J.B. Foresman, J.V. Ortiz, J. Cioslowski, D.J. Fox, Gaussian 09, Revision A.1, Gaussian, Inc., Wallingford CT, 2009.

[29] D.R. Cooper, B. D’Anjou, N. Ghattamaneni, B. Harack, M. Hilke, A. Horth, N. Majlis, M. Massicotte, L. Vandsburger, E. Whiteway, V. Yu, Experimental review of graphene, ISRN Condens. Matter Phys. 2012 (2012) 501686-1-501686-56, , https:// doi.org/10.5402/2012/501686.

[30] J. Wen, Y. Dong, Y. Wen, Q. Liu, H. Wang, Q. Wang, Adsorption of graphene on an $\mathrm{Fe}_{3} \mathrm{O}_{4}$ surface: a molecular dynamics simulation study, J. Adhes. 94 (2017) 238-253, https://doi.org/10.1080/00218464.2016.1260453.

[31] P.F. Guan, X.F. Zhang, J.J. Guo, Assembled $\mathrm{Fe}_{3} \mathrm{O}_{4}$ nanoparticles on graphene for enhanced electromagnetic wave losses, Appl. Phys. Lett. 101 (2012) 153108, https://doi.org/10.1063/1.4758931.

[32] J. Zhou, H. Song, L. Ma, X. Chen, Magnetite/graphene nanosheet composites: interfacial interaction and its impact on the durable high-rate performance in lithiumion batteries, RSC Adv. 1 (2011) 782-791.

[33] H. Atci, Y. Polat, M. Huseyinoglu, B. Arikan, A. Siddiki, DFT modelling of the eff ;ect of strong magnetic field on aniline molecule, arXiv:1611.00552 [cond-mat.meshall].

[34] A. Mishra, V.K. Singh, T. Mohanty, Coexistence of interfacial stress and charge transfer in graphene oxide-based magnetic nanocomposites, J. Mat. Sci. 52 (2017) 7677-7687, https://doi.org/10.1007/s10853-017-1062-1. 